\title{
Desenvolvimento de sobremesas diet e light e sua inclusão no cardápio de uma unidade de alimentação e nutrição
}

\author{
Development of diet and light desserts and their inclusion in the menu of a food service
}

\author{
Gabriela Gelbcke ${ }^{1}$ \\ Ana Carolina Fernandes ${ }^{2}$ \\ Taita Salua Lima Carballo $0^{3}$ \\ 1 Graduanda em Nutrição. Universidade Federal \\ de Santa Catarina. Florianópolis, SC. \\ ${ }^{2}$ Nutricionista e Mestre em Nutrição pela \\ Universidade Federal de Santa Catarina. \\ Professora Substituta do Departamento de \\ Nutrição da Universidade Federal de Santa \\ Catarina (UFSC). Núcleo de Pesquisa de Nutrição \\ em Produção de Refeições (NUPPRE - UFSC). \\ ${ }^{3}$ Nutricionista graduada pelo Centro Universitário \\ Metodista - IPA.
}

Correspondência / Correspondence Ana Carolina Fernandes

E-mail:ana.fernandes@ufsc.br

\section{Resumo}

Diante do atual panorama de doenças crônicas, diversos produtos diet e light já existem no mercado para uma população em torno de 30 milhões de consumidores, em sua maioria diabéticos, obesos, hipertensos, hiperlipidêmicos, hipercolesterolêmicos e demais consumidores. Observa-se, porém, a falta de opções diet e light em muitas Unidades de Alimentação e Nutrição (UAN). Assim, objetivou-se desenvolver sobremesas diet e light para compor o cardápio de uma UAN. Realizou-se pesquisa de receitas em páginas da web e em receituários de outras unidades, avaliando-se custo, variedade, tempo de preparo e aceitação das sobremesas. Foram aprovadas sete sobremesas: duas reduzidas em gorduras, quatro sem sacarose e reduzidas em gorduras, e uma sem lactose. Assim, foi possível elaborar sobremesas mais saudáveis para grupos especiais, e demonstrar a viabilidade de produção de sobremesas diet e light preservando padrão sensorial e com custos acessíveis.

Palavras-chave: Sobremesas. Diet. Light. Unidade de Alimentação e Nutrição.

\section{Abstract}

In today's scenario of chronic diseases, many diet and light products are already on the market for a population around 30 million consumers, mostly diabetics, obese, hypertensive, hyperlipidemic, hypercholesterolemic, and others. There is, however, the lack of diet and light options in a Food Service (FS). Thus, the objective is to develop diet and light desserts to make up 
a menu of FS. Recipes were researched on web pages and other units, assessing cost, variety, preparation time and acceptance of the desserts. Seven desserts were approved: two reduced in fat, four without sucrose and reduced in fat, and one without lactose. Thus it was possible to prepare healthier desserts for special groups, and demonstrate the feasibility of production of diet and light desserts preserving sensory patterns and with affordable costs.

Key words: Desserts. Diet. Light. Food Service.

\section{Introdução}

Estudos recentes têm demonstrado que o consumo habitual da dieta ocidental, caracterizada por uma alta ingestão de carnes vermelhas, produtos lácteos integrais, bebidas adocicadas, açúcares e sobremesas, está diretamente relacionado ao risco de desenvolver obesidade, doenças cardiovasculares (FUNG et al., 2001) e diabetes (GITTELSOHN et al., 1998).

O rápido crescimento mundial do consumo de alimentos processados, amparado em sofisticadas estratégias de marketing desenvolvidas pelas indústrias multinacionais que controlam o setor, é uma das causas importantes da epidemia global de obesidade, diabetes e outras doenças crônicas. Esses alimentos ultraprocessados tendem a apresentar concentrações de gordura, açúcar e sal excessivas e prejudiciais à saúde (WHO, 2003).

Diante do atual panorama de doenças crônicas, centenas de produtos diet e light já existem à disposição no mercado, para uma população em torno de 30 milhões de consumidores, entre os quais se encontram em maioria os diabéticos, obesos, hipertensos, hiperlipidêmicos, hipercolesterolêmicos e consumidores que, por opção, desejam manter uma boa condição de saúde, manter o peso e uma boa aparência física (VILELA, 2000).

Segundo a Anvisa (1998), os produtos light são indicados para o consumo de grupos populacionais saudáveis, muito embora não haja a necessidade de sua utilização para obtenção de uma dieta saudável (BRASIL, 2006). Já os produtos diet são indicados para grupos populacionais com necessidades específicas.

O alimento light é classificado deste modo quando apresenta baixo teor ou teor reduzido de algum nutriente e/ou valor energético com relação a um alimento de referência do mesmo grupo (ANVISA, 1998). Já o alimento diet é assim classificado quando há ausência de determinado componente, como açúcar, sal, glúten, etc. Isso não significa a redução do valor calórico do alimento em questão (VIEIRA; CORNELI, 2007).

Além de produtos industrializados, essa demanda abrange também as Unidades de 
Alimentação e Nutrição (UAN) que, para atingirem seus objetivos de promotoras da saúde, devem oferecer preparações equilibradas nutricionalmente e de acordo com os critérios de qualidade sensorial de seus comensais (AMORIM et al., 2005), incluindo preparações diet e light para a clientela com necessidades dietéticas específicas.

Neste contexto, e buscando também atender a solicitações de comensais, o presente estudo objetivou desenvolver sobremesas com teores reduzidos de gordura e sem açúcar para uma UAN onde não existiam tais opções.

\section{Metodologia}

A pesquisa de receitas envolveu a coleta de dados em sites especializados, em livros técnicocientíficos, em fichas técnicas de preparação (FTP) de receitas tradicionais da UAN e em receitas desenvolvidas em estudo anterior para outra UAN da mesma rede (KARL, 2009). As informações coletadas foram adaptadas para a realização dos testes, sendo alterados e/ou substituídos ingredientes para tentar caracterizar as preparações como diet/light, bem como para adequá-las às porções utilizadas e ingredientes disponíveis no local. Para a seleção das receitas, foram considerados os critérios de custo, tempo de preparo, variedade de receitas e grupos (diet e light).

A avaliação da adequação das receitas de acordo com a classificação diet e light teve como referência a classificação disposta pela Anvisa (1998), conforme especificado:
Gorduras totais: baixo ou light: máximo de $3 \mathrm{~g} / 100 \mathrm{~g}$ da preparação. Reduzido: redução mínima de $25 \%$ com relação ao alimento de referência. A diferença deve ser maior que $3 \mathrm{~g} / 100 \mathrm{~g}$.

Açúcar (sacarose ou lactose): baixo ou light: máximo de $5 \mathrm{~g} / 100 \mathrm{~g}$ e máximo de 40 $\mathrm{kcal} / 100 \mathrm{~g}$ da preparação. Diet: máximo 0,5g do dissacarídeo de referência/100g da preparação, ou máximo $0,5 \mathrm{~g}$ de gordura total $/ 100 \mathrm{~g}$ da preparação (ANVISA, 1998).

Analisou-se o custo de produção das sobremesas desenvolvidas através de pesquisa de preço dos ingredientes com os fornecedores da unidade e em outros estabelecimentos. As receitas que apresentaram valores adequados ao padrão da UAN foram aprovadas para o teste.

As receitas foram testadas na cozinha da unidade e as porções de cada sobremesa foram definidas de acordo com o padrão da UAN, assim como a apresentação da preparação - com o registro fotográfico, que fez parte da composição da FTP. Foram elaboradas etiquetas de identificação contendo o nome da preparação, a classificação (diet, light ou diet e light), especificando o nutriente ausente e/ou reduzido em questão: sacarose (açúcar), lactose ou lipídio (gorduras totais).

A avaliação de aceitabilidade foi realizada no dia da finalização de cada receita por funcionários da cozinha da unidade, assim como por funcionários administrativos da mesma empresa. Estes receberam porções da sobremesa a ser avaliada, a ficha de 
avaliação e instruções de como realizar a avaliação, adotando-se os critérios: "ótimo", "bom", "regular", "ruim" e "péssimo". Foi utilizada a ficha de avaliação adaptada de Isensee (2008), método que descreve "sabor", "textura" e "aparência" das preparações como quesitos a serem avaliados. Os resultados foram tabulados e avaliados para verificar a aprovação da receita ou necessidade de alterações. Foram consideradas aceitáveis as receitas que apresentaram somatório maior que 50\% nos critérios "bom" e "ótimo" para todos os quesitos. Em um primeiro momento, não foi possível realizar testes com os demais comensais, mas no local há a possibilidade de se comunicar sugestões por escrito.

Os dados coletados durante os testes das receitas foram utilizados para a construção de fichas técnicas de preparação, sendo adotado o modelo de FTP (utilizando o programa Microsoft Excel 2003) já existente para as outras preparações do local (PUDLA; SOUZA, 2010).

O valores referentes a energia, carboidratos, proteínas, lipídios e sódio foram calculados utilizando-se a informação nutricional contida no rótulo de produtos ou a Tabela Brasileira de Composição de Alimentos (NEPAUNICAMP, 2006). Quando não encontrados nessa referência, os dados foram retirados da Tabela de Composição de Alimentos de Philippi (2002). Na construção das FTPs, foram avaliados os aspectos nutricionais que deveriam ter destaque - que no caso das sobremesas são as classificações quanto a quantidade de lipídios e açúcar e presença ou não de lactose.
Os valores de sódio ( $\mathrm{Na}$ ) foram avaliados utilizando critérios adaptados da Food Standards Agency (FSA-UK), que traz a classificação de alto, médio e baixo teores de sal em $100 \mathrm{~g}$ de alimentos. Para tanto, esses valores de sal foram convertidos para o correspondente em sódio, obtendo-se a classificação: alto - mais de $600 \mathrm{mg}$ de $\mathrm{Na} / 100 \mathrm{~g}$ da preparação; médio - entre 120 e $600 \mathrm{mg}$ de $\mathrm{Na} / 100 \mathrm{~g}$ da preparação; e baixo - abaixo de $120 \mathrm{~g}$ de Na/100g da preparação.

\section{Resultados e discussão}

Foram desenvolvidas sete sobremesas diet e light para compor o cardápio da unidade, a saber: manjar de coco light (redução de gordura e valor calórico), morango com brigadeiro light (redução de gordura), mousse de limão diet e light (redução de gordura e valor calórico, sem sacarose), pudim de abacaxi diet e light (redução de gordura, sem sacarose), creme de chocolate diet (sem lactose), mousse de maracujá diet e light (redução de gordura e valor calórico, sem sacarose), e gelado de morango com ricota diet e light (redução de gordura, sem sacarose).

As sobremesas foram elaboradas na UAN, e o tempo de preparo foi compatível com o horário de produção. Foram então desenvolvidas as FTPs, padronizadas para o rendimento de 20 porções cada. $\mathrm{O}$ peso das porções foi determinado considerando-se o volume da embalagem padrão, o tamanho das porções de sobremesas já existentes no local e o custo por porção. A lista de ingredientes para 20 porções de cada sobremesa desenvolvida encontra-se descrita no quadro 1. 
Quadro 1. Lista de ingredientes para 20 porções de cada sobremesa desenvolvida

\begin{tabular}{|c|c|}
\hline $\begin{array}{l}\text { Nome da Preparação, } \\
\text { rendimento e peso da porção }\end{array}$ & Ingredientes e quantidades \\
\hline $\begin{array}{l}\text { Manjar de coco light } \\
20 \text { porções de } 110 \mathrm{~g}\end{array}$ & $\begin{array}{l}\text { Leite desnatado (1,5 l), leite condensado light (360 } \\
\mathrm{g}) \text {, leite de coco light (330 g), amido de milho (120 g), } \\
\text { ameixa seca ( } 60 \mathrm{~g}) \text {, coco ralado desengordurado e não } \\
\text { adoçado ( } 50 \mathrm{~g}) \text {, adoçante culinário (10 g) }\end{array}$ \\
\hline $\begin{array}{l}\text { Morango com } \\
\text { brigadeiro light } \\
20 \text { porções de } 120 \mathrm{~g}\end{array}$ & $\begin{array}{l}\text { Morango }(630 \mathrm{~g}) \text {, leite condensado light }(610 \mathrm{~g}) \text {, leite } \\
\text { desnatado }(290 \mathrm{ml}) \text {, creme de leite light }(270 \mathrm{~g}) \text {, chocolate } \\
\text { em pó }(70 \mathrm{~g}) \text {, amido de milho }(50 \mathrm{~g}) \text {, açúcar }(30 \mathrm{~g})\end{array}$ \\
\hline $\begin{array}{l}\text { Mousse de Limão diet e light } \\
20 \text { porções de } 90 \mathrm{~g}\end{array}$ & $\begin{array}{l}\text { Suco de limão (1,8 l), iogurte natural ( } 1,5 \mathrm{l}) \text {, adoçante } \\
\text { culinário (200 g), gelatina incolor sem sabor ( } 40 \mathrm{~g})\end{array}$ \\
\hline $\begin{array}{l}\text { Pudim de abacaxi diet e light } \\
20 \text { porções de } 110 \mathrm{~g}\end{array}$ & $\begin{array}{l}\text { Abacaxi }(2,04 \mathrm{~kg}) \text {, leite desnatado }(1,8 \mathrm{l}) \text {, pudim de } \\
\text { baunilha diet }(120 \mathrm{~g})\end{array}$ \\
\hline $\begin{array}{l}\text { Creme de chocolate diet } \\
\text { (sem lactose) } \\
20 \text { porções de } 80 \mathrm{~g}\end{array}$ & $\begin{array}{l}\text { Creme de soja }(800 \mathrm{~g}) \text {, chocolate em pó (600 g), açúcar } \\
(400 \mathrm{~g}) \text {, morango* }(120 \mathrm{~g}) \text {, essência de baunilha }(40 \mathrm{~g}) \text {, } \\
\text { uva* (40 g) }\end{array}$ \\
\hline $\begin{array}{l}\text { Mousse de maracujá diet } \\
\text { e light } \\
20 \text { porções de } 110 \mathrm{~g}\end{array}$ & $\begin{array}{l}\text { Suco concentrado de maracujá ( } 3,27 \text { l), iogurte natural } \\
(1,36 \mathrm{l}) \text {, polpa natural de maracujá }(250 \mathrm{~g}) \text {, adoçante } \\
\text { culinário }(230 \mathrm{~g}) \text {, gelatina incolor sem sabor }(20 \mathrm{~g})\end{array}$ \\
\hline $\begin{array}{l}\text { Gelado de morango com } \\
\text { ricota diet e light } \\
20 \text { porções de } 125 \mathrm{~g}\end{array}$ & $\begin{array}{l}\text { Água (1,25 l), morango (620 g), iogurte natural (500 } \\
\text { ml), ricota fresca ( } 250 \mathrm{~g}) \text {, leite em pó desnatado ( } 200 \mathrm{~g}) \text {, } \\
\text { gelatina diet sem sabor ( } 30 \mathrm{~g}) \text {, adoçante culinário (50 g) }\end{array}$ \\
\hline
\end{tabular}

* Frutas utilizadas para decoração

As sobremesas que tiveram porções de menor peso foram a mousse de limão diet e light, para manter o padrão de volume da versão original da sobremesa, e o creme de chocolate diet sem lactose, por ter o custo e a densidade energética mais elevados. A porção de maior peso foi a de gelado de morango com ricota diet e light.
Tendo em vista a elaboração das FTPs com as porções definidas, foi possível avaliar a quantidade de energia, macronutrientes e sódio por $100 \mathrm{~g}$ e por porção das sobremesas (tabela 1).

Conforme exposto na tabela 1, todas as sobremesas, com exceção do creme de chocolate diet (sem lactose), possuem menos de $3 \mathrm{~g}$ de lipídios por $100 \mathrm{~g}$, conforme definido pela 
Anvisa para ser considerado um alimento light. Pode-se também verificar que, dentre todas as sobremesas desenvolvidas, o pudim de abacaxi diet e light contém menos calorias, proteínas e lipídios por porção. O creme de chocolate diet sem lactose possui a porção mais calórica, com maior quantidade de lipídios e carboidratos por ser a única receita que contém açúcar e também pelo creme de soja, que contém mais calorias e lipídios.

Avaliando-se o sódio, observou-se que todas as sobremesas apresentaram baixos teores, de acordo com a classificação adaptada da FSA (2009), mesmo se utilizando alguns produtos dietéticos compostos por edulcorantes ricos nesse nutriente. Observou-se, também, que

Tabela 1. Quantidade de energia, proteínas, lipídios, carboidratos e sódio por 100g e por porção das sobremesas diet e light desenvolvidas. Florianópolis, SC, 2010.

\begin{tabular}{|c|c|c|c|c|c|}
\hline $\begin{array}{c}\text { Preparação } \\
\text { Por } 100 \mathrm{~g} \text { e Por Porção }\end{array}$ & $\begin{array}{l}\text { Energia } \\
\text { (Kcal.) }\end{array}$ & $\operatorname{PTN}(g)$ & $\operatorname{LIP}(g)$ & $\mathrm{CHO}(\mathrm{g})$ & \\
\hline \multicolumn{6}{|l|}{ Manjar de coco light } \\
\hline Por $100 \mathrm{~g}$ & 100,5 & 3,8 & 2,9 & 14,9 & 88,1 \\
\hline Por Porção (110g) & 103,8 & 4,2 & 3,2 & 15,0 & 96,95 \\
\hline \multicolumn{6}{|l|}{ Morango com brigadeiro light } \\
\hline Por $100 \mathrm{~g}$ & 123,7 & 3,8 & 1,9 & 22,8 & 48,8 \\
\hline Por porção (120g) & 149,8 & 4,6 & 2,4 & 27,5 & 58,57 \\
\hline \multicolumn{6}{|l|}{ Mousse de limão diet e light } \\
\hline Por $100 \mathrm{~g}$ & 125,8 & 5,6 & 2,6 & 20,0 & 94,3 \\
\hline Por porção $(90 g)$ & 111,8 & 4,7 & 2,3 & 18,0 & 84,89 \\
\hline \multicolumn{6}{|l|}{ Pudim de abacaxi diet e light } \\
\hline Por $100 \mathrm{~g}$ & 92,8 & 3,3 & 0,1 & 19,7 & 91,7 \\
\hline Por porção (110g) & 81,6 & 3,6 & 0,1 & 21,7 & 100,9 \\
\hline \multicolumn{6}{|l|}{ Creme de chocolate diet $\mathrm{s} /$ lactose } \\
\hline Por $100 \mathrm{~g}$ & 333,9 & 4,4 & 12,8 & 50,2 & 13,2 \\
\hline Por porção (80g) & 267,0 & 3,5 & 10,3 & 40,2 & 10,65 \\
\hline \multicolumn{6}{|l|}{ Mousse de maracujá diet e light } \\
\hline Por $100 \mathrm{~g}$ & 148,8 & 4,5 & 2,4 & 27,3 & 52,2 \\
\hline Por porção (110g) & 163,2 & 5,0 & 2,6 & 29,9 & 57,54 \\
\hline \multicolumn{6}{|c|}{ Gelado de morango com ricota diet e light } \\
\hline Por $100 \mathrm{~g}$ & 71,1 & 5,1 & 1,5 & 9,3 & 60,7 \\
\hline Por porção (125g) & 86,5 & 6,4 & 1,9 & 11,7 & 75,99 \\
\hline
\end{tabular}


os ingredientes que mais contribuíram com o sódio das preparações foram os laticínios.

Desta maneira, o trabalho desenvolvido foi ao encontro do recomendado na Diretriz 6 do Guia Alimentar para a População Brasileira (BRASIL, 2006), que diz respeito ao setor produtivo e à necessidade de incentivar a redução de gorduras, açúcares e sal dos alimentos produzidos.

Em relação ao custo, a preparação de valor mais elevado por porção foi o creme de chocolate diet (sem lactose), por conter ingredientes mais caros, como o creme de soja e o chocolate em pó. O gelado de morango com ricota diet e light foi a preparação de menor custo, pois além do baixo custo dos ingredientes, apresentou bom rendimento.

O resultado do teste de aceitação em percentual pelos funcionários do local está exposto na tabela 2. Ressalta-se que os critérios "ruim" e "péssimo" não foram citados por nenhum avaliador.

Tabela 2. Resultados do teste de aceitação em percentual das sobremesas diet e light com colaboradores da unidade produtora de refeições. Florianópolis,SC, 2010.

\begin{tabular}{|c|c|c|c|c|c|c|c|c|c|}
\hline \multirow[t]{2}{*}{ Preparação } & \multicolumn{3}{|c|}{ SABOR } & \multicolumn{3}{|c|}{ TEXTURA } & \multicolumn{3}{|c|}{ APARÊNCIA } \\
\hline & Ótimo & Bom & Regular & Ótimo & Bom & Regular & Ótimo & Bom & Regular \\
\hline $\begin{array}{l}\text { Manjar de coco } \\
\text { light }\end{array}$ & 40 & 55 & 5 & 55 & 42 & 3 & 55 & 39 & 6 \\
\hline $\begin{array}{l}\text { Morango com } \\
\text { brigadeiro light }\end{array}$ & 35 & 60 & 5 & 35 & 65 & 0 & 35 & 65 & 0 \\
\hline $\begin{array}{l}\text { Mousse de limão } \\
\text { diet e light }\end{array}$ & 13 & 77 & 10 & 31 & 56 & 13 & 50 & 50 & 0 \\
\hline $\begin{array}{l}\text { Pudim de abacaxi } \\
\text { diet e light }\end{array}$ & 41 & 46 & 13 & 31 & 56 & 13 & 46 & 46 & 8 \\
\hline $\begin{array}{l}\text { Creme de } \\
\text { chocolate diet s/ } \\
\text { lactose }\end{array}$ & 54 & 33 & 13 & 47 & 46 & 7 & 67 & 33 & 0 \\
\hline $\begin{array}{l}\text { Mousse de } \\
\text { maracujá diet e } \\
\text { light }\end{array}$ & 60 & 35 & 5 & 50 & 45 & 5 & 70 & 30 & 0 \\
\hline $\begin{array}{l}\text { Gelado de } \\
\text { morango c/ ricota } \\
\text { diet e light }\end{array}$ & 80 & 12 & 8 & 45 & 45 & 10 & 78 & 22 & 0 \\
\hline
\end{tabular}


A sobremesa com maior aprovação de sabor e aparência foi o gelado de morango diet e light, enquanto o manjar de coco light se destacou pela textura. Os critérios "ruim" e "péssimo" não foram preenchidos por nenhum dos avaliadores. Desta maneira, considerandose o critério regular, as sobremesas menos aceitas no quesito textura foram a mousse de limão diet e light e o pudim de abacaxi diet e light. No quesito sabor, o creme de chocolate diet sem lactose e o pudim de abacaxi diet e light apresentaram as menores aceitações. No quesito aparência, o pudim de abacaxi diet e light também recebeu a maior porcentagem no critério regular, com $8 \%$, sendo esta a sobremesa com menor aprovação entre todas as desenvolvidas. Segundo as observações das avaliações realizadas, esta menor aprovação se deve, principalmente, ao gosto residual do adoçante presente na mistura para pudim diet utilizada.

As disparidades na avaliação das receitas podem estar relacionadas ao padrão alimentar presente no grupo que realizou a avaliação, pois a maioria relatou que consome diariamente alimentos muito açucarados. Desta forma, sugere-se expandir a avaliação aos demais comensais do local, que também dispõem de um canal de comunicação de sugestões por escrito no refeitório da UAN.

Ainda assim, o resultado foi positivo para todas as avaliações realizadas e todas as receitas desenvovidas nesse período foram aprovadas, pois todas tiveram aceitação bastante acima do ponto de corte estabelecido. Resultados semelhantes foram encontrados por Klemba et al. (2007), quando os avaliadores, ao provarem gelatina sabor morango em versões original, diet e light, aprovaram as versões com ausência de açúcar e reduzidas em calorias, sendo até mesmo constatada a preferência por essas versões. No estudo de Mercer et al. (2008) também houve aceitação acima do esperado de sobremesas diet e light, apesar de os provadores não terem o hábito de consumir sobremesas nessas versões.

\section{Conclusões}

No presente estudo, foi possível desenvolver sobremesas com redução de gorduras e isenção de açúcar, sem elevar o sódio, conforme necessidade da unidade e solicitação dos comensais, sendo aprovadas também no teste de aceitação realizado pelos colaboradores, clientes cativos do local. Destaca-se, porém, a necessidade de realizar avaliação com os demais comensais do local.

Com a inserção destas novas receitas, também foi reduzida a utilização excessiva de cremes de confeitaria e misturas pré-prontas ricas em gordura hidrogenada e açúcar refinado, possibilitando assim o atendimento dos princípios de uma alimentação saudável. Por fim, foi possível demonstrar a viabilidade de se produzir opções mais saudáveis para o público, sem alterar o custo de produção nem o padrão sensorial das sobremesas já oferecidas. 


\section{Referências}

AMORIM, M.M.A; JUNQUEIRA, R.G; JOKL, L. Adequação nutricional do almoço self-service de uma empresa de Santa Luzia, MG. Rev. de Nutrição, Campinas, n.18, v.1, p.145-156, jan./fev., 2005.

BRASIL. Ministério da Saúde. Secretaria de Vigilância Sanitária. ANVISA. Aprovação Regulamento Técnico referente à Informação Nutricional Complementar. Portaria nº 27, de 13 de janeiro de 1998.

BRASIL. Ministério da Saúde. Coordenação-Geral da Política de Alimentação e Nutrição. Guia alimentar para a população brasileira: promovendo a alimentação saudável. Brasília: Ministério da Saúde, 2006.

FOOD STANDARDS AGENCY OF UNITED KINGDOM. The little book of Salt. England: FSA, 2009. Disponível em: http://www.food.gov.uk/ multimedia/pdfs/saltbook1009.pdf. Acesso em: 08 mar 2012.

FUNG, T.T.; RIMM, E.B.; SPIEGELMAN, D.; RIFAI, N.; TOFLER, G.H.; WILLETT, W.C.; HU, F.B. Association between dietary patterns and plasma biomarkers of obesity and cardiovascular disease risk. American Journal of Clinical Nutrition, v.73, p.61-67, 2001.

GITTELSOHN, J.; WOLEVER, T.M.S.; HARRIS, S.B.; HARRIS-GIRALDO, R.; HANLEY, A.J.G.; ZINMAN, B. Specific patterns of food consumption and preparation are associated with diabetes and obesity in a native Canadian community. Journal of Nutrition, v.128, p.541-547, 1998.

ISENSEE, M. Treinamento culinário para redução do teor de gordura e estabelecimento de padrão mínimo de qualidade nutricional e sensorial das preparações da unidade de alimentação e nutrição do Hotel SESC Cacupé. Relatório de Estágio Supervisionado em Administração em Serviço de Alimentação. Florianópolis: Departamento de Nutrição, Universidade Federal de Santa Catarina, 2008.
KARL, J. Padronização de receitas e elaboração de Fichas Técnicas de Preparação de sobremesas na Unidade Produtora de Refeições do Hotel SESC-Cacupé. Relatório de Estágio Supervisionado em Administração em Serviço de Alimentação. Florianópolis: Departamento de Nutrição, Universidade Federal de Santa Catarina, 2009.

KLEMBA, E.A.; DOBRZANSKI, J.; GOMES, D.D.; JUNIOR, G.S. Análise sensorial de gelatina diet, light e convencional de morango, v.2, n.1. Ponta Grossa: Universidade Tecnológica Federal do Paraná, 2125 maio 2007.

MERCER, E.N.; NASCIMENTO, G.; CORREIA, V.A.; SOARES, V.L. Desenvolvimento e produção de um mousse de goiaba com posterior avaliação sensorial, v.2, n.1. Ponta Grossa: Universidade Tecnológica Federal do Paraná, 2008.

NEPA-UNICAMP. Tabela Brasileira de Composição de Alimentos. 2 ed. Campinas: NEPAUNICAMP, 2006.

PEREIRA, G.F. Construção de instrumento para auxiliar na elaboração de cardápios para a Unidade de Alimentação e Nutrição SESC - Prainha. Relatório de Estágio Supervisionado em Administração em Serviço de Alimentação. Florianópolis: Departamento de Nutrição, Universidade Federal de Santa Catarina, 2009.

PHILIPPI, S.T. Tabela de Composição de Alimentos: suporte para decisão Nutricional. 2 ed. São Paulo: Coronário, 2002.

PUDLA, K.J.; SOUZA, M.T. Elaboração de fichas de preparação de alguns pratos principais e das sopas servidas no Restaurante Comerciário SESC - Prainha. Relatório de Estágio Supervisionado em Administração em Serviço de Alimentação. Florianópolis: Departamento de Nutrição, Universidade Federal de Santa Catarina, 2010. 
VIEIRA, A.C.P.; CORNÉLIO, A.R. Produtos lighte diet: o direito de informação ao consumidor. Revista Jurídica Eletrônica, n.45, ano X, set. 2007.

VILELA, E. R. Alimentos dietéticos e alternativos. Textos Acadêmicos. Lavras: UFLA, 2000.
WORLD HEALTH ORGANIZATION. Diet, nutrition and the prevention of chronic diseases. Report of a Joint WHO/FAO Expert Consultation. Geneva: WHO, 2003 (WHO Technical Report Series 916).

Recebido: 11/9/2011

Aprovado: 25/2/2012 